METROLOGY AND MEASUREMENT SYSTEMS
Index 330930, ISSN 0860-8229
www.metrology.pg.gda.pl

\title{
MEASUREMENTS OF SPECTRAL SPATIAL DISTRIBUTION OF SCATTERING MATERIALS FOR REAR PROJECTION SCREENS USED IN VIRTUAL REALITY SYSTEMS
}

\author{
Adam Mazikowski, Michal Trojanowski \\ Department of Metrology and Optoelectronics, Faculty of electronics, Telecommunications and Informatics, Gdańsk University \\ of Technology, Narutowicza 11/12,80-233 Gdańsk, Poland ( $\triangle$ adamazik@eti.pg.gda.pl, +48 606715 540)
}

\begin{abstract}
Rapid development of computing and visualisation systems has resulted in an unprecedented capability to display, in real time, realistic computer-generated worlds. Advanced techniques, including three-dimensional (3D) projection, supplemented by multi-channel surround sound, create immersive environments whose applications range from entertainment to military to scientific. One of the most advanced virtual reality systems are CAVE-type systems, in which the user is surrounded by projection screens. Knowledge of the screen material scattering properties, which depend on projection geometry and wavelength, is mandatory for proper design of these systems. In this paper this problem is addressed by introducing a scattering distribution function, creating a dedicated measurement setup and investigating the properties of selected materials used for rear projection screens. Based on the obtained results it can be concluded that the choice of the screen material has substantial impact on the performance of the system.
\end{abstract}

Keywords: scattering distribution function, BSDF, virtual reality, CAVE, spectroscopy, luminance.

(C) 2013 Polish Academy of Sciences. All rights reserved

\section{Introduction}

Rapid development of computers and visualisation systems allows the developers to concentrate on creating very realistic computer-generated worlds, called virtual reality (VR). To achieve the best quality and "feeling" of VR, the combination of three basic elements is needed: interaction with the generated world, ability to move freely and a perception of depth. These three elements are also referred in literature as $\mathrm{I}^{3}$ : Interaction + Immersion + Imagination [1]. All of those elements are being introduced in computer games where players can easily "sink into" their virtual world by having the ability to interact with almost every element of the displayed scene. Visual effects are also becoming increasingly realistic making them difficult to distinguish from the real world. Some systems allow the player to use the whole body as a controller which enhances his interaction with VR.

An important limitation of most display systems used in the computer game environment is the size and extent of the generated image: only one screen, sometimes composed of multiple monitors, is used to display the scene, restricting his view to the space in front of the user. Several classes of current and potential applications of VR, e.g.:

- military (for training pilots, paratroopers, equipment operators),

- medicine (for training surgeons, dentists),

- entertainment and tourism, 
require screens with three-dimensional (3D) projection which cover the space around the user. Geometrical configuration of these projection systems makes them vulnerable to scattering, which will be discussed later in this paper. Knowledge of the spatial scattering distribution function of screen materials is mandatory for proper design of these systems. Since this information is not readily available, an experimental setup had to be developed in order to perform measurement of this function. Using this setup, the scattering properties of selected, commonly used projection screen materials were measured.

\section{Virtual reality systems}

The first virtual reality systems began to appear in the late twentieth century due to rapid development of computers. One of first systems was called CAVE which stands for Cave Automatic Virtual Environment. It was built at the University of Illinois at Chicago in 1992 $[2,3]$. It consisted of a cube in "classic" configuration (Fig.1a) in which four of the walls acted as projection screens. Projection was usually onto the floor and on three walls, one in front and two on the sides of the user. Projection on the ceiling was available as an option. However, an important disadvantage of such implementation of the CAVE is lack of complete immersion of the user in the projected scene, due to the lack of projection on the fourth wall and on the ceiling. The ability to move around is also limited due to the small size of the cube (approximately $3 \mathrm{~m} \times 3 \mathrm{~m} \times 3 \mathrm{~m}$ ).
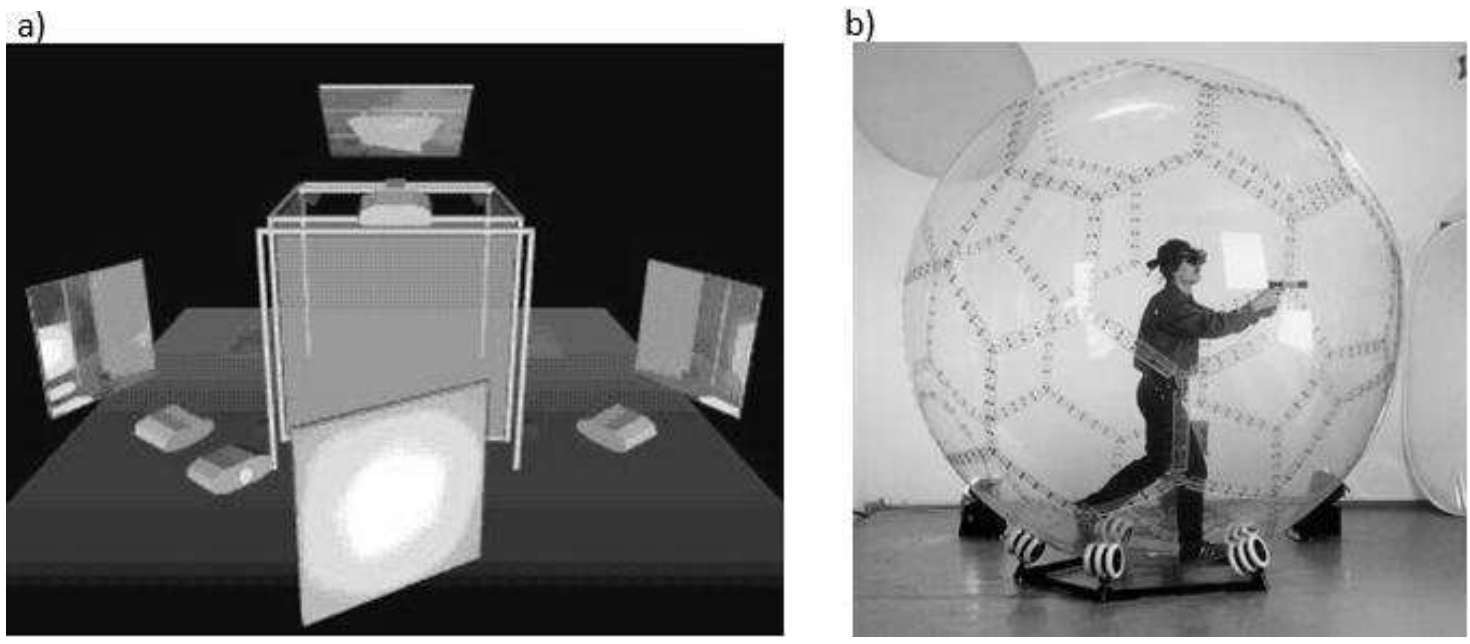

Fig. 1. Examples of virtual reality systems: a), ,classic” CAVE [2], b) virtusphere [4].

To overcome movement restrictions another type of system, called virtusphere, was developed [4] (Fig.1b). It consists of a human-sized ball (sphere) which is mounted on a set of rolls that allows the ball to rotate freely. As a result ,the user who is placed inside the sphere, has a feeling of unrestricted movement [6]. In this type of system, generation of the VR is performed by a cybernetic helmet, resulting in the user's discomfort due to considerable weight of the helmet. There is also a possibility of delay in the projected images in the case of rapid movement of the user, e.g. when he suddenly starts to run or turn back [5]. Even with miniaturization of the cybernetic helmet this solution is not ideal.

Another improvement to the VR system was made in an installation created at the University of Warwick (England) [6] (Fig.2). It comprized a rotating sphere placed in a vertical air stream causing the ball to "float". The sphere was made of two layers of polycarbonate, where each of them consisted of several specially designed shapes [6] in order to ensure mechanical stiffness and provide a spherical projection screen by making the outer layer out of brushed material. This solution has some disadvantages. The most important is 
attaining proper depth of focus for projection on a spherical screen. Another one is difficulty in seamless joining of pictures projected on the sphere from multiple projectors [7].

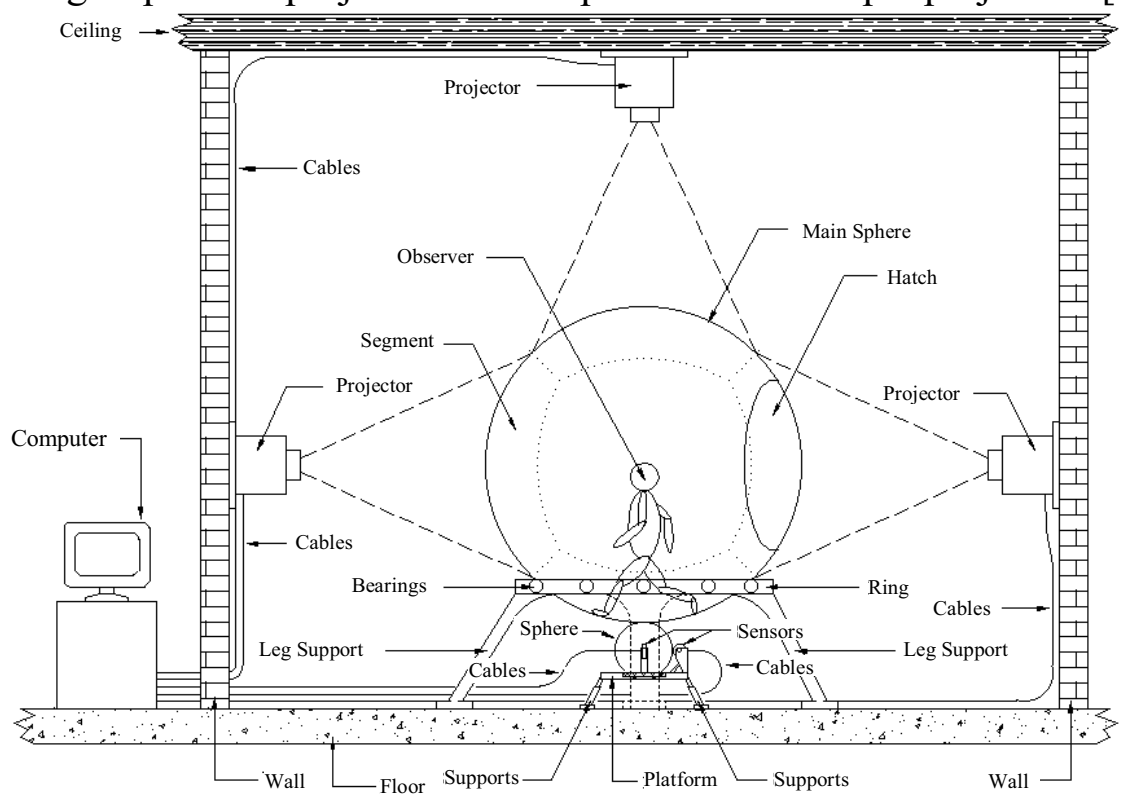

Fig. 2. The virtual reality installation at the University of Warwick [6].

In order to create a VR system better than those presented above, some solutions present in both systems can be implemented. The combined system would consist of a CAVE and a human-sized ball (sphere) which would be placed inside. The sphere must be transparent and stiff to allow a user to be placed inside it and see the image projected on screens. Projection would take place on all sides of the cube (floor, ceiling, walls) giving the advantage of generating VR in every direction that the user can look to while the ball allows the user to have the feeling of free movement.

Such solution is being developed in the Faculty of Electronics, Telecommunications and Informatics of Gdańsk University of Technology. One of key problems is obtaining a homogenous luminance distribution on rear projection screens (walls of the cube). It is determined mostly by properties of the material from which the screens are made and by characteristics of the projectors. Proper joining of projected pictures and pixel size have a direct impact on the quality of VR experienced by the user. The best solution is that the cube must be large enough to be able to put the eyes of the user near the centre of the whole system. To further increase the feeling of being "immersed" in VR, three-dimensional (3D) projection should be applied.

There are several systems of 3D projection. The most common are stereoscopic systems that come in two types: active with projection separated in time (active stereo) and passive with projection separated in polarization (passive stereo) [8].These two methods have one disadvantage while the combined system is developed. The commercially available rotating sphere placed inside is made from a polymer material which may polarize or depolarize light in an uncontrolled manner thus the use of those systems may not work properly. However, a study of another rotating sphere, that will be able to keep polarization will be carried out in the future. Another approach to 3D projection is the stereoscopic method with separation of the spectrum. It is derived of the flaw that the previously mentioned systems had. This system is based on a technology called "wavelength triplet" developed by Infitec [9]. The principle of this system is to separate three wavelengths corresponding to three basic stimuli (colors) seen 
by the human eye (blue, green, red) for the left and right eye respectively. The idea of the system is illustrated in Fig. 3.

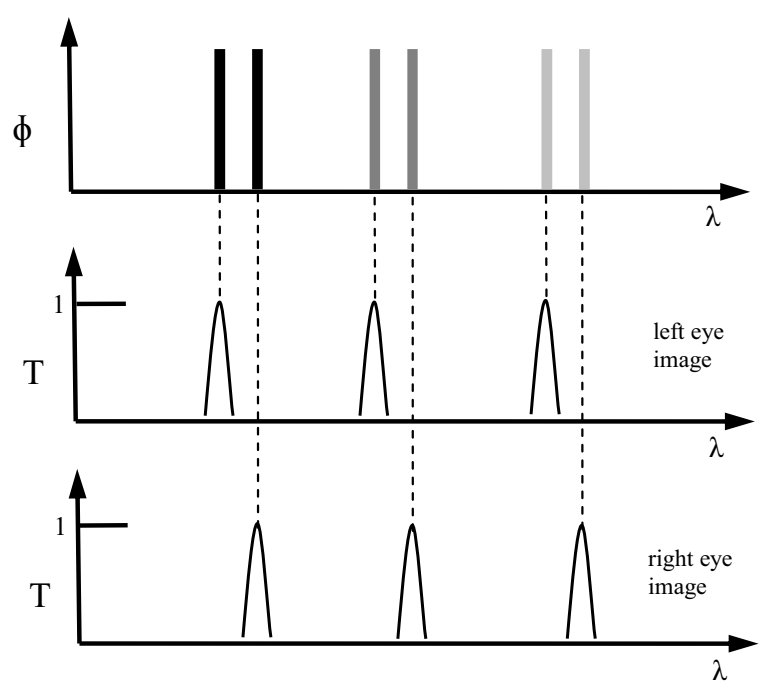

Fig. 3. Characteristic of the light beam projector $\boldsymbol{\Phi}(\lambda)$ and transmission characteristic of filters respectively for the left and right eye [9].

By combining those three primary colors in an additive manner we can obtain any other color. Also we can combine different colors (with different wavelength distribution) to obtain as the result the same color and this is called the metamerism phenomenon [10]. It is used by Infitec to generate two images for each eye that have a small shift in wavelength between each other. Provided that the wavelength shift is small, colors seen by the left and right eye are almost the same (indistinguishable in practice). To use this technology the user must wear special lightweight glasses with a set of interference filters whose transmission characteristic are shown in Fig. 3.

A number of problems of VR systems are still unsolved, both on technical matters and the subjective user perception of $3 \mathrm{D}$ impression as well as the impact on the users health and wellbeing [11].

\section{Optical system requirements}

One of the most important parts of the CAVE-type VR system is the optical system - a system of 3D rear projection. A few types of stereoscopic systems were developed [8]. However, while developing a CAVE-type VR system we need to take a deeper look into the geometry of projection and observation angles. Let us consider the geometry configuration of the classic CAVE (Fig. 4a), projection onto the rotating sphere (Fig. 4b) and a system consisting of classic CAVE with rotating sphere placed inside (such as one being developed at Gdańsk University of Technology) [5].

The classic CAVE system is the worst case scenario of the requirements for projection geometry. The user can move freely on the floor of the CAVE, so the angle of view $\alpha$ (angle of scattered light, incident to user eyes, Fig. 4) can be extremely high. Because of lack of space outside the CAVE, the throw ratio is about 1:1 (this means that dimension of the screen is equal the distance between the screen and the projector) therefore throw angle $\beta$ (angle of incident light from the projector, Fig. 4) is also high. The difference of these angles (difference between directions of projection and viewing) can become even more than $90^{\circ}$. 
Additionally, due to the small distance of the viewer from the screen, pixels seen by the user can be distinguished, so high resolution of projection is required.

Projection onto the sphere requires covering the sphere surface by special scattering layers which provide the projection surface. Assuming that eyes of the user are in a fixed position, about in the middle of the sphere, the viewing angle $\alpha$ is about $0^{\circ}$ (Fig.4.b). However, in this case the throw angle $\beta$ can reach extremely high values.

In the case of a rotating sphere placed inside the classic CAVE, we assume that the eyes of the user are also in a fixed position about in the middle of the sphere and CAVE. The viewing angles cannot reach as high values as in the classic CAVE (the user is in a fixed position), while the throw angle is the same. The difference of view and throw angles can also reach significant values.
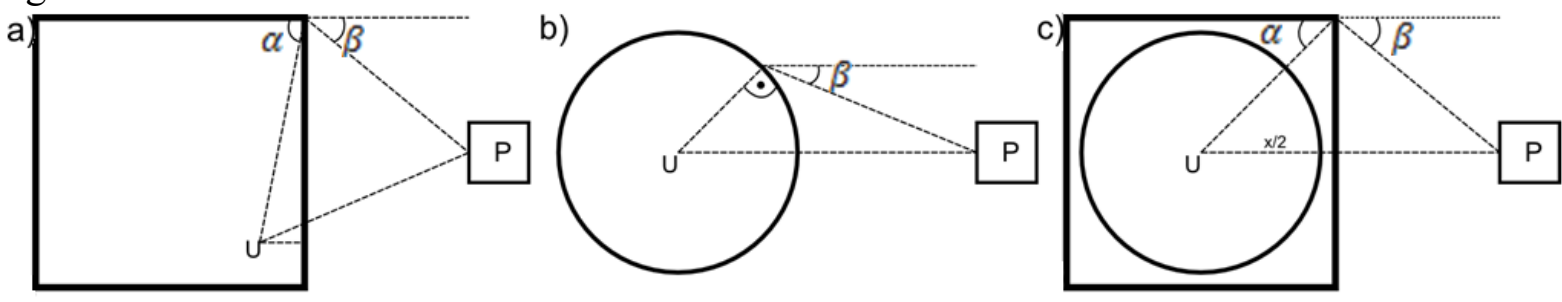

Fig. 4. Geometry of observation and projection in the plane of user sight for three VR systems: $\mathrm{P}$ - projector, $\mathrm{U}-$ user.

High values of projection (throw) and observation (view) angles may cause problems when high quality $3 \mathrm{D}$ projection is needed. First, it may cause luminance differences depending on these angles. Further on the luminance distribution can vary for different wavelengths. It may cause differences of white balance of the image. This problem is very important especially for a rotating sphere placed inside the CAVE, as the best technique for $3 \mathrm{D}$ projection is projection with separation of the spectrum.

Some of these negative effects can be partially compensated by the use of special software and optics. However, technical specification and research concentrate mainly on scattering characteristics for normal throw angle or for narrow range of angles [12, 13], while knowledge of spectral scattering properties of the screen material for high values of projection and observation angles (wide range) is required. This problem may be addressed by measuring or modeling of the scattering distribution function of screens for rear projection, known as BTDF (bidirectional transmittance distribution function) [13, 14], taking into account the geometry of the CAVE-type system.

\section{Measurement setup}

The developed measurement setup corresponds to combining configuration of the system, when the rotating sphere is placed inside the classic CAVE. The maximum viewing angle in that case was calculated as $45^{\circ}$, while the maximum throw angle is evaluated as $24^{\circ}$ (application of projector optics of throw ratio about 1:1 is assumed). For such constraints a measuring setup capable of illuminating the sample at an incident angle up to $40^{\circ}$ and able to measure a dispersed rays cone up to $56^{\circ}$ has been created. The scheme of the setup is presented in Fig. 5a, while the ready-to-use setup is shown in Fig 5b. Samples are placed on a rotating table allowing to get information about scattered rays in the XY plane. The accuracy of setting illumination (throw) angle (rays from the source to the sample) is up to $1^{\circ}$. As a detector a Konica Minolta CS-2000A Spectroradiometer was used. It was mounted on a tripod stand with pivoting head, capable of setting the observation angle with a resolution up to $2^{\circ}$. 

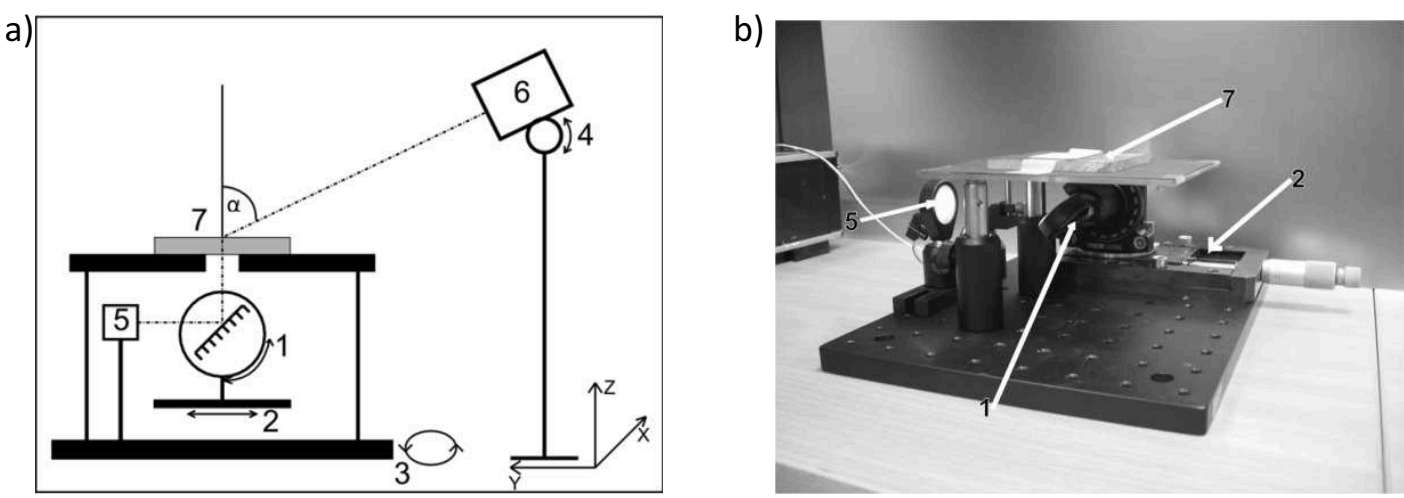

Fig. 5. Scattering measurement setup a), and its photograph b) 1 - mirror on table rotating in the $Z$ axis, 2 - sliding table in the $\mathrm{Y}$ axis, 3 - rotating table in the XY plane, 4 - rotating mount for spectrometer, 5 - light source, 6 - spectrometer, 7 - sample, $\alpha$-throw (illuminance) angle.

The measurement system presented in Fig. 5 is capable to test samples within calculated angle ranges. The photograph of the assembled measurement system ready to use is shown in Fig. 6.

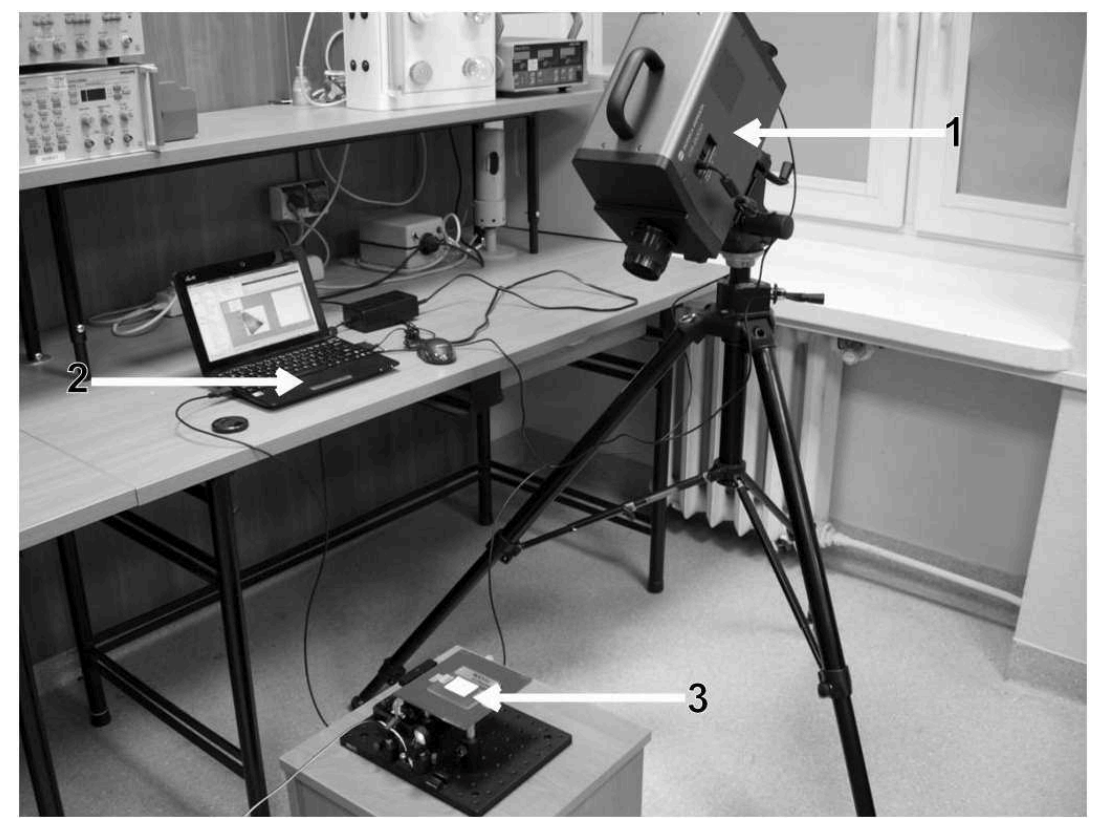

Fig. 6. Scattering measurement system: 1 - spectroradiometer CS-2000A, 2 - computer with dedicated software, 3 - measurement setup.

\section{Measurement results}

Using the measurement setup presented in Fig. 5 and 6, measurements of scattering distribution functions were performed for selected materials. Spatial (angular) distribution functions of screen luminance were measured for two selected materials, Plexiglas RP offered by Evonik Rohm for rear projection [12]. Two materials were selected, the first one PLEXIGLAS ${ }^{\circ}$ OPTICAL 7D006 RP with $45 \%$ transmission, with the scattering layer on both surfaces and 5mm thick, the second one PLEXIGLAS®OPTICAL $99561 \mathrm{RP}$ with 45\% transmission, with the scattering layer on both surfaces and $3 \mathrm{~mm}$ thick. 


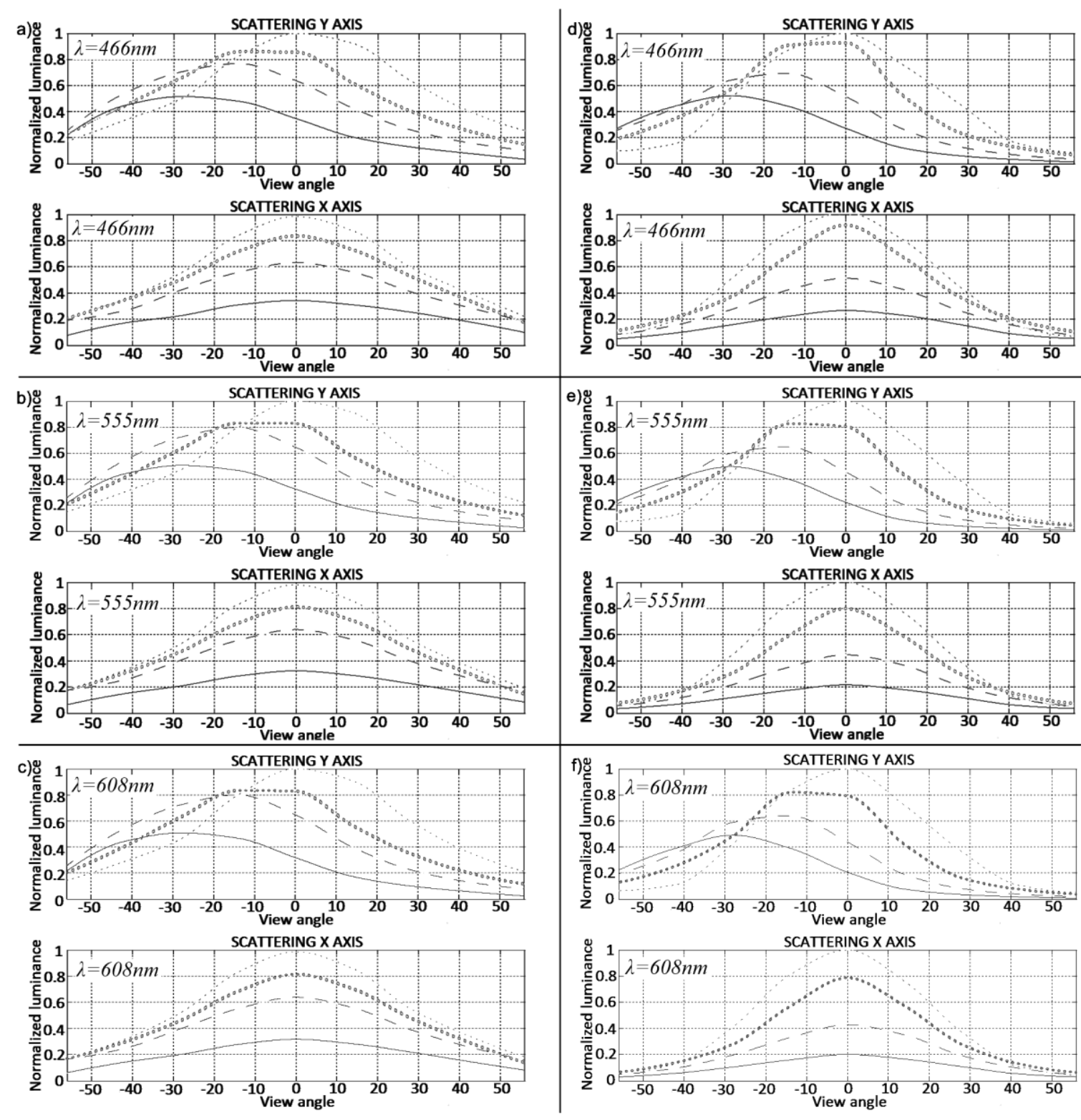

Fig. 7. Measured scattering function for various materials - 7D006RP (Figure a, b and c) and 99561RP (Figure d, $\mathrm{e}$ and $\mathrm{f}$ ), for various wavelength and illuminance angles

$\left(0^{\circ}\right.$ - dot line, $-10^{\circ}$ - circle line, $-20^{\circ}$ - dashed line, $-30^{\circ}$ - solid line $)$.

Measurements were performed for three wavelengths: $466 \mathrm{~nm}, 555 \mathrm{~nm}, 608 \mathrm{~nm}$. Those wavelengths are corresponding to the dominant wavelength of three basic colors (blue, green, red) in a typical projector. Measurements (luminance) were taken as a function of the viewing angle for a few illuminance (throw) angles for two axes $\mathrm{X}$ and $\mathrm{Y}$ (corresponding with the coordinates marked on the measurement setup scheme, Fig. 5.). As a light source a white LED was applied. Results are presented in Fig. 7.

The normalized luminance function is not symmetrical for the $\mathrm{Y}$ axis (changes of illuminance angle were along the $\mathrm{Y}$ axis). Based on obtained results it is possible to assess the normalized luminance for high projection and view angles (e.g. projection angle $-30^{\circ}$, view angle $+50^{\circ}$, the difference of the sum of these angles is $\left.80^{\circ}\right)$. For a thick material $(5 \mathrm{~mm})$ the luminance distribution is flatter than for a thin material $(3 \mathrm{~mm})$ for both $\mathrm{X}$ and $\mathrm{Y}$ axes. Flatter distribution is better for rear projection (from the standpoint of luminance distribution). Additionally, a more detailed analysis shows that luminance distribution changes for different 
wavelengths. For better readability, based on Konica Minolta CS-2000A spectroradiometer software, for the $\mathrm{Y}$ axis there were also calculated color coordinates xy for the measured light - white point (according to the CIE 1931 standard) [15]. They are presented in Fig. 8 for 7D006 RP and in Fig. 9 for 99561 RP respectively. Each of figures consists of four subfigures named a-d. For example Fig. 8a, shows color coordinates xy for all measured viewing angles and illuminance angle of $0^{\circ}$ for 7D006 RP. These figures show that the white point moves through the chromaticity diagram for different view angles. The distance between the extreme points can be considered as a quality parameter of the screen, taking into account changes of light chromaticity (for other CIE standards $\Delta E$ may be introduced). As a reference the white point is used for normal $\left(0^{\circ}\right)$ projection and view angles. This point is also marked (with a cross) in Fig. 8. and Fig. 9.
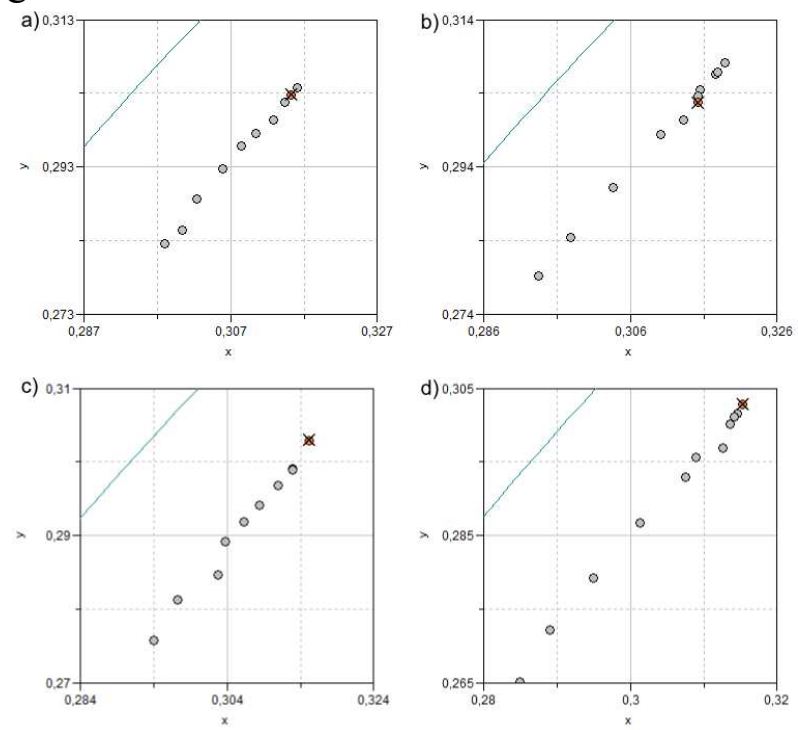

Fig. 8. Color coordinates xy (CIE 1931) for Plexiglas 99561 RP, 3mm thickness, for all view angles: a) illuminance angle $0^{\circ}$, b) illuminance angle $-10^{\circ}$, c) illuminance angle $-20^{\circ}$, d) illuminance angle $-30^{\circ}$.
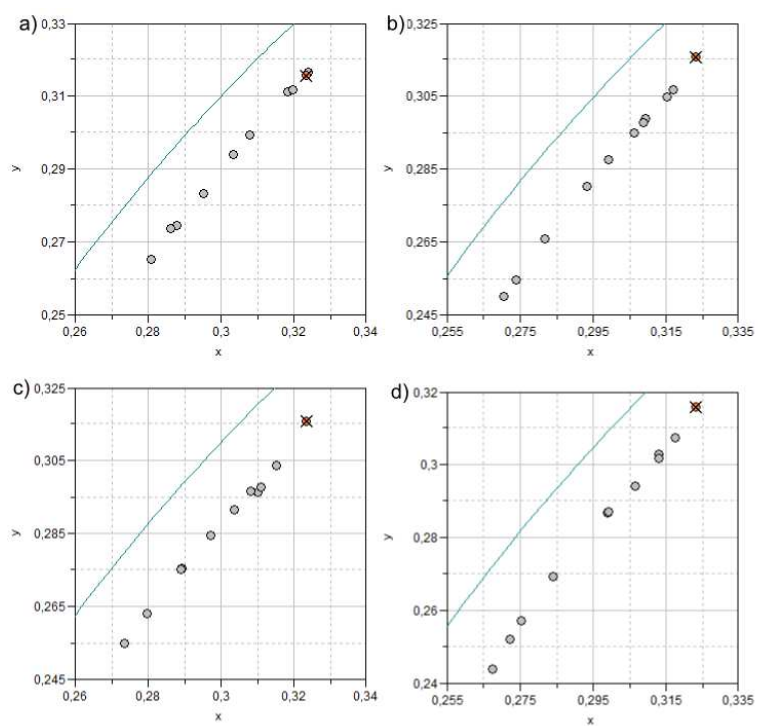

Fig. 9. Color coordinates xy (CIE 1931) for Plexiglas 7D006 RP, 3mm thickness, for all view angles: a) illuminance angle $0^{\circ}$, b) illuminance angle $-10^{\circ}$, c) illuminance angle $-20^{\circ}$, d) illuminance angle $-30^{\circ}$. 
Measurement uncertainty has two main sources: a) uncertainty in setting the incident and observation angles ( $1^{\circ}$ and $2^{\circ}$, respectively) and b) the uncertainty of determination of the chromaticity coordinates $( \pm 0.002$ for the CS-2000A spectroradiometer used in this research [15]).

\section{CONCLUSIONS}

Successful implementation of a virtual reality system with 3D projection is a very complex task, requiring the solution of several problems of different nature: mechanical, optical, control, data processing and display. One of these problems is the dependence of projected 3D scene luminance and color on the angle of observation. It is much more acute in the VR systems than in the systems used in cinemas or TV sets because of different geometry of the former systems.

The dependence of projected 3D scene luminance and color on the angle of observation is determined by the properties of the scattering material used in the projection screen. While knowledge of the spatial scattering distribution function of screen materials is important for design of the $3 \mathrm{D}$ systems, it is not readily available. This necessitates the measurement of the scattering properties of these materials. We expect that some additional techniques to the presented 3D projection like atmoshere compositon monitoring could be applied as well [16].

In this paper a measurement setup for determination of selected scattering (luminance and color) properties of screen materials has been developed. Using this system, the measurements were performed for two materials (differing in thickness). The obtained results show that thicker $(5 \mathrm{~mm}) \mathrm{RP}$ plexiglas has better properties: flatter luminance distribution and less dispersion of coordinates of the white point.

\section{References}

[1] Burdea G., Coiffet, P. (2003). Virtual Reality Technology. New York: $2^{\text {nd }}$ Ed., Wiley

[2] Cruz-Neira C., Sandin D. J., DeFanti T. A. (1992). The CAVE: A Virtual Reality Theatre, HPCCV Publications, 2, Available online from http://www.evl.uic.edu/pape/CAVE/ [September 99].

[3] DeFanti T. A., at al. (March 2011). The future of the CAVE. Central European Journal of Engineering, , 1(1), 16-37.

[4] Medina E., Fruland Weghorst R.S. (2008). Virtusphere: Walking in a Human Size VR Hamster Ball. Proc. of the Human factors and Ergonomics Society Annual Meeting. 52(27), 2102-2106.

[5] Lebiedź J., Łubinski J., Mazikowski A. (June 2010). Immersive 3D Visualization Laboratory Concept. Proc. of the $2^{\text {nd }}$ International Conference on Information Technology, ICIT 2010. Gdańsk, Poland, 28-30.

[6] Fernandes K.J., Raja V., Eyre J. (September 2003). Cybersphere: The Fully Immersive Spherical Projection System. Comm. of the ACM, 46(9), 141-146.

[7] Shulgin B.V., Ye J., Raja V.H. (2006). Multiprojector image distortion correction scheme for curved screens on the example of eh Cybersphere. Proc. SPIE: Stereoscopic Displays and Virtual Reality Systems XIII, 6055.

[8] Stereoscopic projection. 3D projection Technology. Barco: www.vr.barco.com

[9] Jorke H., Fritz M. (2005). Infitec - a new stereoscopic visualization tool by wavelength multiplex imaging. Journal of Three Dimensional Images, 19(3), 50-56.

[10] Wyszecki G., Stiles W.S. (1982). Colour Science: Concepts and Methods, Quantitative data and Formulae. Wiley \& Sons. 
[11] Zieliński J., Olifierczuk M. (2010). 3D Display - Technical conditions of implementation and psychophysical image reception. VI Scientific Symposium: Image Processing Techniques TPO’2010. Warsaw, Poland, 365-373.

[12] Plexiglas RP. High quality rear projection. Evonik Rohm GmbH: http://corporate.evonik.de

[13] Brown A.M., at al. (September 2010). Optical material characterization through BSDF measurement and analysis. Proc. SPIE, Reflection, Scattering and Diffraction from Surfaces II, 7792.

[14] Cassarly W.J. (2011). Using the on-axis BSDF at a dielectric surface to model the BSDF at offaxis angles. Proc. SPIE, Illumination Optics II, 8170

[15] Spectroradiometer CS-2000/CS-2000A Instruction Manual, Konica Minolta: http://sensing.konicaminolta.us

[16] Kwan C., Schmera G., Smulko J. M., Kish L. B., Heszler P., Granqvist C. G. (2008). Advanced agent identification with fluctuation-enhanced sensing. Sensors Journal, IEEE, 8(6), 706-713, DOI: 10.1109/JSEN.2008.923029. 